duration ("on-time") to ITI ("off-time"). PubMed and SCOPUS databases were searched through March 30, 2015 using the terms: "transcranial magnetic stimulation", "TMS", "rTMS", “inter-train interval", "inter-stimulus interval", and "cortical spread". Three hundred and one articles were identified comprising a total of 3359 patients. Clinical outcomes were reported as the proportion of patients achieving response defined as $50 \%$ reduction in baseline score on the primary outcome measure in each study. Treatment risk was assessed by the frequency of adverse events reported, and specifically considering the incidence of seizures.

RESULTS: This analysis confirms prior reports that the variables which impact treatment efficacy are the number of treatmentsessions, the number of pulses per session and the percent motor threshold. Varying the train duration/ITI (on-time/off-time) ratio over a broad range from 2.0 to 14 did not impact efficacy or safety.

CONCLUSIONS: Shortening the ITI to 11 seconds does not impact the safety and antidepressant effectiveness of the NeuroStar TMS and would result in shortening of each treatment session from approximately 37.5 to 19 minutes.

FUNDING ACKNOWLEDGEMENTS: Neuronetics, Inc.

161

\section{The NeuroStar Outcomes Registry}

Miriam Mina'; David G. Brock, MD'; W. Scott West, $M D^{3}$; Todd Hutton, $\mathrm{MD}^{4}$; Kenneth P. Pages, $\mathrm{MD}^{5}$; and Timothy J. Kane, $M D^{6}$

${ }^{1}$ Neuronetics, Inc., Malvern, PA

${ }^{2}$ no affiliation, Ambler, PA

${ }^{3}$ ThriveLogic TMS + NeuroHealth, Vanderbilt University, Nashville, TN

${ }^{4}$ Southern California TMS Center, USC Keck School of Medicine, Pasadena, CA

5 TMS of South Tampa, Tampa, FL

${ }^{6}$ Comprehensive Health Systems, PLC, Fisherville, VA

ABSTRACT: The NeuroStar Outcomes Registry

OBJECTIVE: NeuroStar transcranial magnetic stimulation (TMS) is an effective acute treatment for patients with major depressive disorder (MDD). In order to further understand use of the NeuroStar in a clinical setting, Neuronetics has established a patient treatment and outcomes registry to collect and analyze utilization information on patients receiving treatment with the NeuroStar.
METHODS: Individual NeuroStar providers are invited to participate in the registry and agree to provide their deidentified patient treatment data. The NeuroStar has an integrated electronic data management system (TrakStar) which allows for the data collection to be automated. The data collected for the registry include Demographic Elements (age, gender), Treatment Parameters, and Clinical Ratings. Clinical assessments are: Clinician Global Impression - Severity of Illness (CGI-S) and thePatient Health Questionnaire 9-item (PHQ-9). De-identified patient data is uploaded to Registry server; an independent statistical service then creates final data reports.

RESULTS: Over 500 patients have entered the NeuroStar Outcomes Registry since Sept 2016. Mean patient age: $48.0(\mathrm{SD} \pm 16.0)$; 64\% Female. Baseline PHQ-9, mean 18.8 (SD \pm 5.0.) Response/Remission Rate, PHQ-9: 61\%/33\% CGI-S: $78 \% / 59 \%$.

CONCLUSIONS: For the initial 500 patients in the Outcomes Registry, approximately $2 / 3$ patients achieve respond and $1 / 3$ patients achieve remission with an acute course of NeuroStar. These treatment outcomes consistent with NeuroStar open-label study data (Carpenter, 2012). The TrakStar data management system makes large scale data collection feasible. The NeuroStarOutcomes Registry is ongoing, and expected to reach 6000 outpatients from more than 47 clinical sites in 36 months.

FUNDING ACKNOWLEDGEMENTS: Neuronetics, Inc.

\section{2}

\section{Add-on Filgrastim During Clozapine Rechallenge Unsuccessful in Treating Benign Ethnic Neutropenia}

Molly Britton, $M D^{\prime}$; Palanikumar Gunasekar, $M B B S^{2}$; and Vithyalakshmi Selvaraj, $M D^{3}$

${ }^{1}$ Psychiatry resident, Department of Psychiatry, Creighton University School of Medicine, Omaha, NE

${ }^{2}$ Post doctoral fellow, Department of Clinical Translational Science, Creighton University School of Medicine, Omaha, NE

${ }^{3}$ Associate Professor, Department of Psychiatry, Creighton University School of Medicine, Omaha, NE

ABSTRACT: Clozapine is an atypical antipsychotic approved by the Food and Drug Administration for treatment-resistant schizophrenia and also indicated for the reduction in risk of recurrent suicidal behavior in patients with schizophrenia or schizoaffective disorder. The most serious side effect of clozapine treatment is agranulocytosis, which is defined as an absolute 